\title{
Mitigation of stochastic diffusion losses in optimized stellarators
}

\author{
A. V. Tykhyy ${ }^{1}$, Ya. I. Kolesnichenko ${ }^{1}$, Yu. V. Yakovenko ${ }^{1}$, \\ A. Weller ${ }^{2}$, A. Werner ${ }^{2}$ \\ ${ }^{1}$ Institute for Nuclear Research, 03680 Kyiv, Ukraine \\ ${ }^{2}$ Max-Planck-Institut für Plasmaphysik, D-17489 Greifswald, Germany
}

\begin{abstract}
The stochastic diffusion (SD) resulting from collisionless transformations between locally trapped and locally passing orbits may lead to a significant loss of energetic particles from stellarator plasmas (Beidler C D et al 2001 Phys. Plasmas 8 2731). In this work, a method to mitigate the consequences of SD is suggested. The method consists in changing the shapes of the separatrices between the locally trapped and locally passing states so that most separatrices do not cross the plasma boundary, which is achieved with a suitable modification of the magnetic configuration. It is demonstrated analytically that in this way the particle loss because of the SD may be reduced significantly, even though the SD still occurs. In particular, it is possible to protect fast particles inside a certain radius from SD loss. It is shown that a variation of the electric field can also affect the SD loss. The modifications of the magnetic configuration and the electric field required for the mitigation are quantified for a configuration of the stellarator Wendelstein 7-X.
\end{abstract}




\section{Introduction}

It is known that the lack of axial symmetry in stellarators results in a diversity of particle orbits (see, e.g., [1]). Typically, the variation of the magnetic field strength, $B$, along a field line is characterized by two scale lengths in the toroidal angle, $\varphi$. One of them, $\Delta \varphi \sim 2 \pi / N$, where $N$ is the number of the field periods, is the size of the local magnetic wells. The larger scale, $\Delta \varphi \sim 2 \pi / \iota$, where $\iota$ is the rotational transform, is produced by toroidicity (as well as by the interference of different helical harmonics of $B$ ). As a result, the particles with small magnetic moments are passing, i.e., they never change the direction of their longitudinal motion. Usually, there is a fraction of particles which always remain trapped in local magnetic wells (they are particles with large magnetic moments). The particles with intermediate magnitudes of the magnetic moment are transitioning between the locally trapped state and the locally passing (or toroidally trapped) state. In the latter state, they are trapped by the toroidal inhomogeneity of $B$, passing the local magnetic maxima.

A numerical simulation of a fusion reactor based on traditional stellarator concepts [2] have shown that the majority of trapped energetic ions escape from the plasma with characteristic time of the order of the orbital time scale, i.e., much shorter than the thermalization time. It was realized that this fact would mean the loss of a large fraction of fusion-produced alpha particles, which could constitute a serious problem for designing a stellarator reactor. This increased the interest paid to the confinement of energetic ions in stellarators.

As a result, several new concepts of the stellarator design have appeared, which received the common name of "optimized stellarators". In these devices, the orbits of the collisionless motion of the particles are aligned to the flux surfaces. One of the approaches to designing optimized stellarators is quasi-symmetry. The quasi-symmetric stellarators $[3,4,5]$ are designed so that the magnetic field strength possesses a certain symmetry (helical or toroidal) in flux coordinates, which provides the existence of invariants of the particle motion. A more general approach is known under the names of quasi-omnigeneity or quasi-isodynamicity $[4,6]$. It consists in demanding that the level contours of the adiabatic invariants of the particle motion approximately coincide with the flux surfaces (or that the average drift across the flux surfaces approximately vanishes for one bounce, which is the same) and optimizing the magnetic field of the configuration, having this requirement as one of the optimization criteria. In particular, this approach was used in the development of the device Wendelstein 7-X [7] (W7-X) and several conceptual projects of Helias reactors [8], which were designed as W7-X improved and scaled to the reactor size. The so-called "quasi-poloidal" devices [9] exaggerate one of the features of the Wendelstein-type configurations - a large mirror harmonic of the magnetic field (i.e., the harmonic $\propto \exp (i N \varphi)$, where $\varphi$ is the toroidal angle and $N$ is the number of the field periods). Finally, it was found recently that the inward shift of the magnetic axis in the heliotron LHD (Large Helical Device [10]) results in a significant improvement of the particle confinement [11]. The mechanism 
of the improvement in LHD consists in aligning the minima of $B$ along the field lines, which was actually suggested earlier [12] as a way to reach quasi-isodynamicity for deeply trapped particles.

Numerical simulations of the alpha particle behaviour [13] have shown that the alpha particle confinement in optimized configurations is indeed much better than that in traditional stellarators. Most particles in the optimized configurations, including a majority of trapped particles, are confined for times much longer than the characteristic times of the orbital motion. However, it turned out that a considerable fraction of particles are still lost before their thermalization, the main reason of the loss being the stochastic diffusion (SD), i.e., the collisionless diffusion occurring because of the stochastization of the particle motion. Later it was shown that the most important mechanism of the SD in Wendelstein-type configurations, which dominates the particle loss, is the diffusion of the transitioning particles due to radial jumps during the transitions between the locally trapped and locally passing states [14]. This diffusion is similar to the diffusion of charged particles due to repeated trapping and detrapping in a wave with varying amplitude; it was earlier considered for the particles transitioning between the locally trapped and toroidally trapped states in a tokamak with toroidal field ripple (see [15] and references therein). One can expect that this mechanism is important also in other optimized systems in which there is a large fraction of transitioning particles, e.g., in "quasi-poloidal" devices and optimized heliotron configurations.

In this work, we suggest a method of the mitigation of consequences of the SD caused by particle transitions. Namely, we suggest to modify the configuration so that the separatrices separating the regions of the locally passing particles and the locally trapped ones become closed within the plasma. As will be shown below, this results in a decrease of the particle loss caused by the SD, even though the SD still occurs. Although the model magnetic configuration that we consider is relevant to Wendelsteinline stellarators (we consider a standard configuration of W7-X), the main idea of the mitigation is applicable to other configurations as well.

The structure of the work is as follows. In section 2 the model magnetic field and the equation of the separatrices are described. The principal idea of the proposed way to mitigate the consequences of the stochastic diffusion is explained in section 3. Numerical estimates for a configuration of W7-X are given in section 4. Finally, in section 5 the conclusions are presented.

\section{Model of the magnetic field}

We will use Boozer coordinates $(x, \theta, \varphi)$, where $x$ is a flux surface label, $\theta$ the poloidal angle and $\varphi$ the toroidal angle. The radius-like coordinate $x, 0<x<1$, is defined by $\psi=\psi_{a} x^{2}$, where $\psi$ is the toroidal magnetic flux, and $\psi_{a}$ is its value at the plasma boundary. 
We expand the magnetic field strength $B$ in a Fourier series:

$$
B=\bar{B}\left[1+\sum_{\mu \geq 0, \nu} \epsilon_{\mu \nu}(x) \cos (\mu \theta-\nu N \varphi)\right],
$$

where $\bar{B}$ is the average magnetic field strength on the magnetic axis. Here we restrict ourselves to considering a model magnetic field, in which only the toroidal $(\mu=1$, $\nu=0)$, mirror $(\mu=0, \nu=1)$, helical $(\mu=\nu=1)$, and diamagnetic $(\mu=\nu=0)$ harmonics, which are the largest in W7-X and the Helias reactors, are non-zero. For instance, in W7-X configurations [16], these four harmonics are several times larger than the rest of the harmonics, making a dominant contribution to the division of the phase space into the regions of the trapped and passing particles. Further, we approximate these harmonics with linear and quadratic functions of $x$ : $\epsilon_{10} \equiv-\epsilon_{t}=-c_{t} x$, $\epsilon_{11} \equiv-\epsilon_{h}=-c_{h} x, \epsilon_{00} \equiv \epsilon_{0}=c_{0} x^{2}$ and $\epsilon_{01} \equiv \epsilon_{m}=\epsilon_{m 0}+c_{m} x^{2}$, where $c_{m}, c_{h}, c_{t}$, $c_{0}$ and $\epsilon_{m 0}$ are positive constants determined by the particular magnetic configuration. In W7-X these expressions with an appropriate choice of the coefficients are accurate to within a few percent. Then we can write the magnetic field as follows

$$
\frac{B}{\bar{B}}=1+c_{0} x^{2}-c_{t} x \cos \theta+\epsilon_{h m}(x, \theta) \cos [N \varphi+\chi(x, \theta)]
$$

where $\epsilon_{h m}^{2}=\epsilon_{m}^{2}-2 \epsilon_{m} c_{h} x \cos \theta+c_{h}^{2} x^{2}$, and $\chi(x, \theta)=\cos ^{-1}\left[\left(\epsilon_{m}-c_{h} x \cos \theta\right) / \epsilon_{h m}\right]$.

Consider a particle with mass $M$, charge $e$, full energy $W$ and magnetic momentum $\mu_{p}$ in the guiding center approximation. Particle velocity along the magnetic field is given by $v_{\|}^{2}=2\left(W-\mu_{p} B-e \Phi\right) / M$, where $\Phi$ is the electric field potential. We take this potential to be zero at $x=0$. This means that at $x=0$, full energy $W$ is identical to the particle kinetic energy $M v_{\|}^{2} / 2+\mu_{p} B$. In our model magnetic field the expression for $v_{\|}$reduces to

$$
v_{\|}^{2}=2 \mu_{p} \bar{B} \epsilon_{h m}\left\{\kappa^{2}-\sin ^{2}[(\varphi-\chi) / 2]\right\} / M,
$$

where

$$
\kappa^{2}=\frac{\alpha-\phi-\left(c_{0} x^{2}-c_{t} x \cos \theta\right)}{2\left|\epsilon_{h m}\right|}+\frac{1}{2}
$$

$\alpha=W /\left(\mu_{p} \bar{B}\right)-1$ is a pitch-angle variable and $\phi=e \Phi /\left(\mu_{p} \bar{B}\right)$ is the normalized potential of the electric field. If the particle drift across the field lines is sufficiently slow in comparison with the longitudinal motion and $\iota / N \ll 1$, the coordinates $x$ and $\theta$ are almost constant for the time of the particle transit / bounce motion in one field period. Then we can study the motion in $\varphi$ with $x$ and $\theta$ fixed, considering $\varphi$ as a fast variable and $x$ and $\theta$ as slow variables. With respect to the fast motion in $\varphi$, the expression (3) is the velocity of a nonlinear pendulum, and the separatrix between "rotation" (locally passing particles) and "libration" (locally trapped particles) is given by $\kappa^{2}(x, \theta)=1$. The adiabatic invariant associated with the fast longitudinal motion is [17]:

$$
J^{*}= \begin{cases}\sigma \frac{e}{N c} \Psi_{p}+\frac{M}{2 \pi} \int_{0}^{2 \pi / N} \mathrm{~d} \varphi \frac{B_{\varphi}}{B} v_{\|} & \text {for passing particles } \\ \frac{M}{2 \pi} \oint \mathrm{d} \varphi \frac{B_{\varphi}}{B} v_{\|} & \text {for trapped particles }\end{cases}
$$



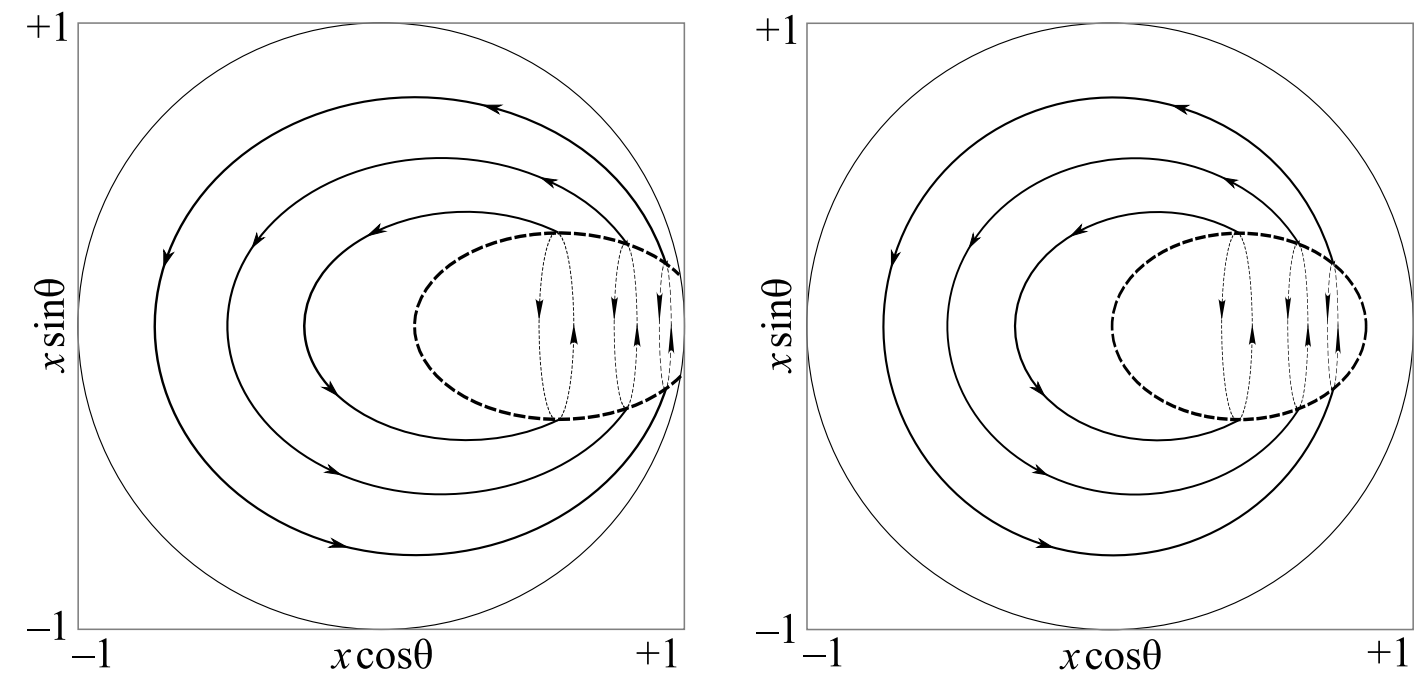

Figure 1. Sketches of two different types of separatrices. Left: the particle leaves the plasma when the transition point wanders randomly along the separatrix. Right: the particle transition point is confined within the plasma. Notation: thin black line, the plasma boundary; bold dashed line; the separatrix; lines with arrows, different orbits of the same particle in the process of stochastic diffusion (the motion in the locally passing state between the reflection points is shown inside the separatrix; the precession motion in the locally trapped state, outside).

where $\sigma=\operatorname{sgn}\left(v_{\|}\right), \Psi_{p}$ is the poloidal magnetic flux, $B_{\phi}$ is the corresponding covariant component of $\boldsymbol{B}$. The integrals in this equation are taken at constant $x$ and $\theta$, the integral for trapped particles being taken between the particle turning points.

\section{The principal idea of the mitigation}

When transitioning particles cross the separatrix $\kappa^{2}(x, \theta)=1$ because of precession in $x$ and $\theta$, they suffer transformations between the locally passing state and the locally trapped state. Because adiabaticity breaks down near the separatrix, the adiabatic invariant $J^{*}$ accumulates random jumps, which lead to stochastic diffusion. The separatrix, though, is completely determined by $W$ and $\mu_{p}$, and when there is no electric field, these two parameters occur in (4) only in the $\alpha$ combination. All three quantities $W, \mu_{p}$ and $\alpha$ are constants of motion in the guiding-centre approximation, and the breakdown of adiabaticity for $J^{*}$ does not influence them. Therefore, in the process of stochastic diffusion a given particle will always cross the same separatrix, even though its orbit (determined by $J^{*}$ in addition to $W$ and $\mu_{p}$ ) changes at each crossing. The crossing point will, naturally, wander along the separatrix.

Observing this, we conclude that not every particle which experiences stochastic diffusion may escape from the plasma. This is demonstrated in figure 1, where the bounce-averaged / transit-averaged particle motion is shown in the poloidal plane. We assume that the random jumps of the particle adiabatic invariants are small, so that at each instant we can characterize the particle by a certain closed orbit, which slowly 
evolves because of the jumps. If the particle's separatrix is closed within the plasma and the bounce-averaged orbits of locally trapped particles are sufficiently close to $x=$ const (figure 1, right), the particle is unable to leave the plasma despite stochastic diffusion. On the other hand, if the separatrix crosses the plasma boundary (figure 1, left), the particle will most likely be lost on the stochastic diffusion time scale unless the slowingdown time is shorter. Note that if the bounce-averaged particle orbit is not closed within the plasma, the particle is lost on the much shorter precession time scale, and in this case it is meaningless to discuss stochastic diffusion at all.

To obtain more concrete results, consider locally trapped particles. For these particles we use the approximation of zero orbit width, $x=$ const. This approximation is justified in optimized stellarators. A particle with given $x$ and $\alpha$ is transitioning if $\kappa^{2}=1$ at some $\theta$. It follows from equation (4) that this condition can be satisfied only for particles with $\alpha$ in a certain range

$$
\alpha_{\min }(x)<\alpha<\alpha_{\max }(x) .
$$

In our model magnetic field the quantities $\alpha_{\min }$ and $\alpha_{\max }$ are determined by solving the equation $\kappa(x, \theta, \alpha)=1$ for $\alpha$ at $\theta=0$ and $\theta=\pi$, respectively, and are given by

$$
\begin{aligned}
& \alpha_{\max }(x)=c_{0} x^{2}+\phi(x)+c_{t} x+\epsilon_{m}+c_{h} x, \\
& \alpha_{\min }(x)=c_{0} x^{2}+\phi(x)-c_{t} x+\left|\epsilon_{m}-c_{h} x\right| .
\end{aligned}
$$

Figure 2 shows two graphs of $\alpha_{\min }(x)$ and $\alpha_{\max }(x)$, for the standard W-7X high-beta configuration and for the same configuration modified by decreasing the mirror harmonic to $c_{m}=0.03$ and $\epsilon_{m}(0)=0$.

Stochastic diffusion losses are markedly lower in the modified configuration. Indeed, the particles are lost only from those separatrices which cross the $x=1$ boundary. A separatrix for particles with a given $\alpha$ is represented on the graphs by a horizontal line segment $x \in\left[x_{\min }(\alpha), x_{\max }(\alpha)\right]$, where the functions $x_{\min }(\alpha)$ and $x_{\max }(\alpha)$ are the inverse functions to $\alpha_{\max }(x)$ and $\alpha_{\min }(x)$, respectively, so that $\alpha_{\max }\left(x_{\min }(\alpha)\right) \equiv \alpha$ and $\alpha_{\min }\left(x_{\max }(\alpha)\right) \equiv \alpha$. The marginal separatrix has $\alpha=\alpha_{\min }(1)$. It divides the "phase space" of transitioning particles $(x, \alpha)$, which is bounded by equation (6) and the inequality $x<1$, into two parts, and particles escape only from the upper part. Therefore, the proportion of the particles that can be lost because of stochastic diffusion increases with the area of the upper part relative to the area of the lower part.

Note that for any choice of the constants in our model magnetic field and for any $\phi(x)$ profile, $\alpha_{\max }(x)$ is strictly greater than $\alpha_{\min }(x)$ for all $x \in(0,1)$. Therefore, some separatrices always cross the plasma boundary, and thus it is impossible to prevent all stochastic diffusion losses.

If for a particle with a given $\alpha$ the largest root $x_{r}$ of the equation $\alpha_{\min }\left(x_{r}\right)=\alpha$ is less than unity, stochastic diffusion cannot lead to the loss of such a particle. From a different point of view, all particles located inside a certain radius $x_{*}$ determined from

$$
\alpha_{\max }\left(x_{*}\right)=\alpha_{\min }(1)
$$



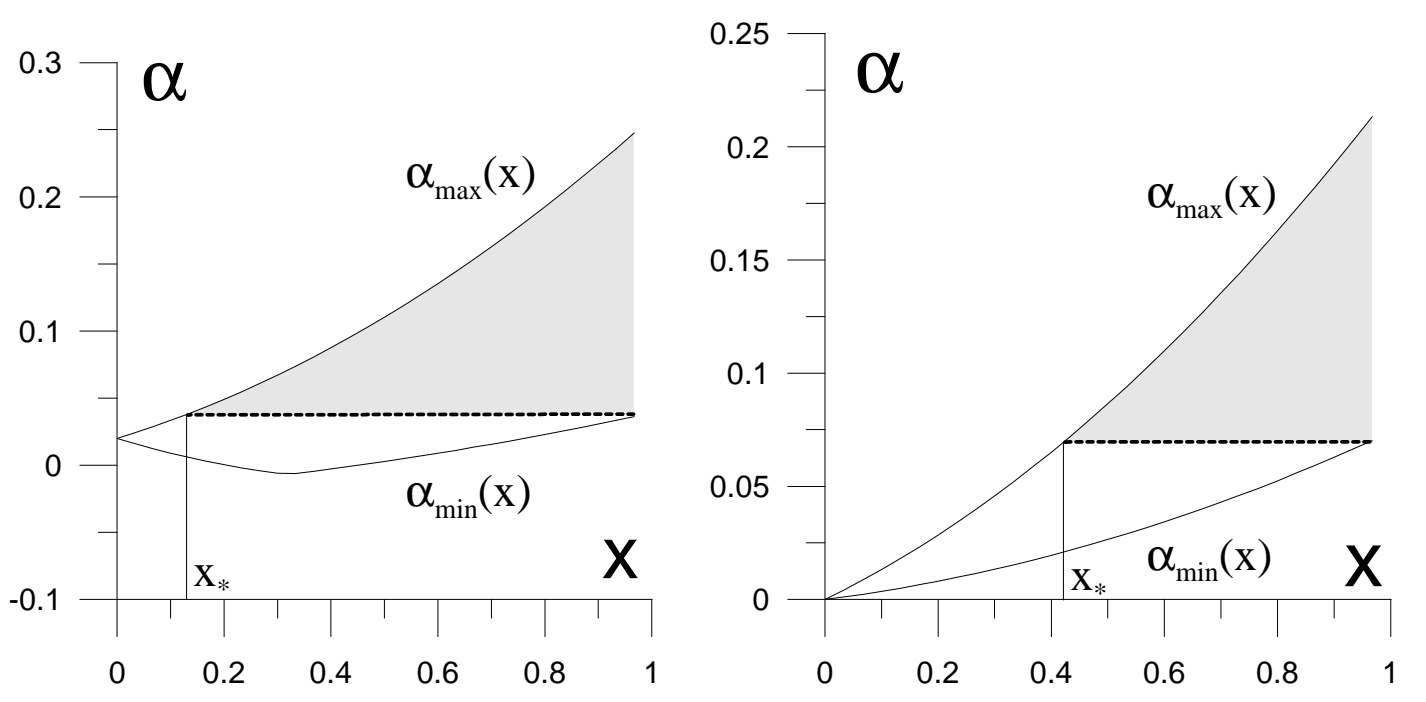

Figure 2. $\alpha_{\min }$ and $\alpha_{\max }$ versus $x$. Left: for standard $\mathrm{W}-7 \mathrm{X}$ high-beta configuration $\left(c_{0}=0.072, c_{m}=0.046, \epsilon_{m}(0)=0.02, c_{t}=0.041, c_{h}=0.077, \beta_{0}=0.094\right.$, where $\beta_{0}$ is the ratio of the plasma pressure to the magnetic field pressure at $x=0$ ) in the absence of the electric field $(\phi=0)$. Right: for the same configuration with decreased mirror harmonic $\left(c_{m}=0.03, \epsilon_{m}(0)=0\right)$. Marginal separatrix, defined by $\alpha=\alpha_{\min }(1)$, shown in heavy dashed line. Stochastic diffusion leads to loss of particles only in the shaded areas.

also cannot be lost, because they either do not cross any separatrix and experience no stochastic diffusion, or their separatrix is completely inside the plasma.

To obtain a solution of (8), we approximate the normalized electric potential with the sum of a quadratic potential, which corresponds to rigid rotation of energetic ions, and a potential barrier of height $\delta \phi_{b}$ with a maximum at $x=1$ such that the barrier is localized outside the radius $x_{*}$ :

$$
\phi(x)=c_{\phi} x^{2}+\delta \phi_{b} f(x),
$$

where $f(x)$ is the barrier shape function with the maximum value $f(1)=1$. Its exact form is not important in our model provided that $f\left(x_{*}\right) \ll 1$, because the electric field potential adds both to $\alpha_{\min }$ and $\alpha_{\max }$. If the barrier is localized at a smaller radius $x_{b}<1$, it affects $x_{*}$ only if $\alpha_{\min }\left(x_{b}\right)+\delta \phi_{b}>\alpha_{\min }(1)$. If this is the case, $x_{*}$ may be found by substituting $x_{b}$ instead of 1 into the right-hand side of (8).

Using (9), we obtain the solution to (8):

$$
x_{*}=-y+\left[(y-1)^{2}+\delta\right]^{1 / 2},
$$

where $y=\left(c_{h}+c_{t}\right) / 2\left(c_{0}+c_{m}+c_{\phi}\right)$ and

$$
\delta=\frac{\left|\epsilon_{h}(1)-\epsilon_{m}(1)\right|+\epsilon_{h}(1)-\epsilon_{m}(1)+\delta \phi_{b}}{c_{0}+c_{m}+c_{\phi}} .
$$

When $\epsilon_{h}(1)<\epsilon_{m}(1)$ and $\delta \phi_{b}=0$, as, e.g., in the high-mirror W-7X configurations, this solution simplifies to

$$
x_{*}=1-\frac{c_{t}+c_{h}}{c_{0}+c_{m}+c_{\phi}} .
$$


We can see that in this case, increasing $c_{0}$ and the parabolic component of the mirror harmonic, $c_{m}$, (e.g., by increasing the plasma pressure) and adding a negative radial electric field increases $x_{*}$ and lessens the stochastic diffusion losses. Note that increasing the constant component of the mirror harmonic, $\epsilon_{m}(0)$, does not improve $x_{*}$. In the opposite case, i.e. when $\epsilon_{h}(1)>\epsilon_{m}(1)$, the influence of the mirror and helical harmonics depends on the relative magnitudes of all harmonics in our model, but larger values of $\epsilon_{m}(0)$ always lead to smaller $x_{*}$.

\section{Numerical estimates}

Let us first consider the high-mirror configuration of $\mathrm{W}-7 \mathrm{X}$ with $\beta_{0}=0.068$ (see, e.g., figure 2 in [16]). Substituting appropriate values for the harmonics of the magnetic field $\left(c_{t}=0.043, c_{h}=0.08, c_{0}=0.05, c_{m}=0.03, \epsilon_{m}(1)=0.125\right)$, we see that the expression (12) is valid in this case. We obtain that $x_{*}<0$, which means that there are no closed separatrices at all. Individual particle orbits are also poorly confined in these configurations. To obtain $x_{*}=0.5$, the denominator of (12) needs to be tripled. If we adopt the reasonable assumption that $c_{0}$ and $c_{m}$ grow linearly with $\beta_{0}$, which is the $\beta$ at $r=0$, and estimate the relationship from magnetic configurations with different $\beta_{0}$, it follows that $\beta_{0}$ in excess of 0.2 is necessary, which is hardly of practical importance in $\mathrm{W}-7 \mathrm{X}$, but may be relevant to a Helias reactor. We can also estimate the radial electric field required to reach the same value of $x_{*}=0.5$. For the case without the barrier $\left(\delta f_{b}=0\right)$, equation (9) yields $c_{\phi} \sim e E_{r}^{\prime} a^{2} / 2 W$. From this estimate we can see that for $50-\mathrm{keV}$ particles in the same high-mirror configuration we would need an electric field on the order of $30 \mathrm{kV} / \mathrm{m}$, corresponding to plasma rotation with the frequency of $1.2 \times 10^{4} \mathrm{~s}^{-1}$. This is much larger than the typical values. Even the combined effects of both negative electric field and plasma pressure is insufficient to significantly improve the situation. Moreover, when $\beta_{0}$ is increased, the overall $\beta$ profile tends to become more peaked, and the approximation of quadratic $x$ dependence in the mirror and diamagnetic harmonics breaks down, invalidating the solution (10).

In the standard configurations of W-7X [16], the inequality $\epsilon_{h}(1)>\epsilon_{m}(1)$ holds, and we must use equation (10). Then we obtain $x_{*} \approx 0$ in the standard configuration with $\beta_{0}=0.068$, but already in the high-beta variant with $\beta_{0}=0.094$ we have $x_{*}=0.17$, which means that the particles in the inner plasma core do not escape through stochastic diffusion. If we modify this configuration, decreasing the mirror harmonic by a third to $c_{m}=0.03$ and $\epsilon_{m}(1)=0.03$ (see figure 2), $x_{*}$ increases to 0.46 . Thus, the stochastic diffusion loss may be reduced by changing the mirror harmonic, which can be easily manipulated in W7-X via variations of the current in the circular coils.

The effect of localized electric fields also must be estimated with (10). In the same standard, high-beta W-7X configuration for a 50-keV particle, a barrier $3 \mathrm{kV}$ high will raise $x_{*}$ to 0.5 . This "electric transport barrier" action of the negative electric field works together with its beneficial effect on individual particle orbits described in [16].

The effect of electric field decreases with particle energy. While for the relatively 
slow 50-keV particles it is stronger than the effect of a magnetic configuration change, for thermonuclear $\alpha$-particles the electric fields necessary to affect particle drift motion materially are quite out of the feasible range. This suggests that a positive electric field may help to remove helium "ash" in a reactor. It was suggested in [16] to use the electric field to deconfine orbits of locally trapped particle of certain energies with this purpose. When the stochastic diffusion time is less than the slowing-down time for cooled-down $\alpha$-particles, opening the separatrices of transitioning particles of low energies may serve the same purpose.

On the other hand, changes of magnetic configuration affect particle confinement regardless of particle energy. In particular, larger $\beta_{0}$ is beneficial for the confinement. In a reactor, this fortuitously agrees with the need of high $\beta_{0}$ for efficient operation.

Since the formalism that we use here does not examine individual particle drift orbits, there is a potential danger that while the separatrix is closed inside the plasma, the contours of the adiabatic invariant which cross this separatrix are not. We checked for this possibility numerically by comparing the sets of values of the adiabatic invariant on the separatrix and at the plasma edge. If these sets do not intersect, no drift orbits are lost from the separatrix. The check shows that such cases, when separatrices are closed while drift orbits are not, are rare.

\section{Conclusions}

A method of stochastic diffusion loss mitigation is suggested in this work. This mitigation is due to the closing of separatrices between locally trapped and locally passing states within the plasma. If, for a given pitch-angle, the particle drift orbits and the separatrix are closed within the plasma, the particles will not escape from the plasma on the stochastic diffusion timescale. If the orbits are not confined, the separatrix shape does not matter. However, in Wendelstein-type stellarators the orbits tend to be confined when the corresponding separatrix is closed within the plasma. Although our consideration disregards slowing down and, therefore, is applicable to the case when the slowing-down time well exceeds the stochastic diffusion time, one can expect that closing the separatrices within the plasma is favourable for the particle confinement in the case when these characteristic times are comparable, too.

It is shown that in Wendelstein-type stellarators, plasma diamagnetism helps to close the separatrices, while the toroidal harmonic tends to make them more open. The role of the mirror and helical harmonics depends on their relative magnitudes. In particular, in the case when the helical harmonic exceeds the mirror one at the plasma boundary, a decrease of the mirror harmonic, which can be controlled by the circular magnetic coils in W7-X, can prevent fast ion loss caused by stochastic diffusion from within almost half the plasma radius.

Variations of the radial electric field (which can happen due to, e.g., transitions between the electron root and the ion root or the formation of a transport barrier) can affect the mitigation. Specifically, the negative radial electric field helps to close 
the separatrices. Localized potential barriers improve the separatrix shape when the negative field region lies inside the positive field region. In the standard high-beta configuration of $\mathrm{W}-7 \mathrm{X}$, an electric potential barrier $3 \mathrm{kV}$ high closes all separatrices of $50-\mathrm{keV}$ particles that intersect the $x=0.5$ boundary, so that the transitioning particles within this boundary cannot escape. In some configurations, positive electric fields, which increase the stochastic diffusion losses rather than decrease them, may be beneficial - for instance, they may help to remove helium "ash" in a reactor. Since the effect of the electric field decreases with the particle energy, this increase of the loss will weakly affect high-energy particles.

If considered in a wider context, magnetic configuration modifications favourable for closing the separatrices within the plasma may happen to be adverse from the point of view of some other criteria of the configuration quality (achievable $\beta$, quasiisodynamicity etc.). Hence, closure of separatrix shapes within the plasma could be added as an additional criterion during the optimization of stellarator configurations. Minimization of fast particle loss calculated with a Monte Carlo code is often included in the set of criteria in the process of the stellarator optimization (see, e.g., [13]). However, closure of separatrix shapes within the plasma is a criterion evaluated much faster than the particle loss with a Monte Carlo code (although, admittedly, the latter criterion takes into account loss channels other than stochastic diffusion of transitioning particles). It should be emphasized that closure of separatrix shapes within the plasma is not the same as the alignment of particle orbits to the flux surfaces (quasi-isodynamicity).

\section{Acknowledgments}

The work is carried out within the Partner Project Agreement No. P-034f between the Science and Technology Center in Ukraine, the Kyiv Institute for Nuclear Research, and the Max-Planck-Institut für Plasmaphysik. One of the authors (Y.Y.) would like to acknowledge the hospitality of the Max-Planck-Institut für Plasmaphysik.

\section{References}

[1] Galeev A A and Sagdeev R Z 1979 Problems of Plasma Theory ed M A Leontovich (New York: Consultants Bureau) vol 7, p 257

[2] Painter S L and Lyon J F 1989 Fusion Technol. 16157

[3] Nührenberg J and Zille R 1988 Phys. Lett. A 129113

[4] Nührenberg J, Lotz W and Gori S 1994 Theory of Fusion Plasmas ed E Sindoni, F Troyon and J Vaclavik (Bologna: SIF) p 3

[5] Garabedian P R 1996 Phys. Plasmas 32483

[6] Cary J R and Shasharina S G 1997 Phys. Rev. Lett. 78674

[7] Nührenberg J et al 1995 Trans. Fusion Technol. 2771

[8] Wobig H et al 2003 Nucl. Fusion 43889 and references therein

[9] Strickler D J et al 2004 Fusion Sci. Technol. 4515

[10] Fujiwara M et al 2001 Nucl. Fusion 411355

[11] Komori A et al 2003 Plasma Phys. Control. Fusion 45671

[12] Mynick H E et al 1982 Phys. Rev. Lett. 48322 
[13] Lotz W and Nührenberg J 1992 Theory of Fusion Plasmas ed E Sindoni and J Vaclavik (Bologna: SIF) p 17

[14] Beidler C D et al 2001 Phys. Plasmas 82731

[15] Marchenko V S 1995 Nucl. Fusion 3569

[16] Kolesnichenko Ya I et al 2006 Phys. Plasmas 13072504

[17] Cary J R, Hedrick C L and Tolliver J S 1988 Phys. Fluids 311586 